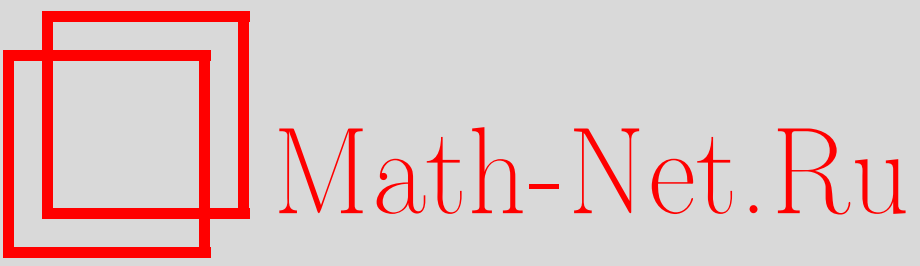

Л. А. Калякин, Возмущение решений с подвижными особыми точками, ТМФ, 2001, том 127, номер 3, 401-410

DOI: https://doi.org/10.4213/tmf468

Использование Общероссийского математического портала Math-Net.Ru подразумевает, что вы прочитали и согласны с пользовательским соглашением

http://www.mathnet.ru/rus/agreement

Параметры загрузки:

IP: 54.89 .56 .158

26 апреля 2023 г., 18:17:16 
ТЕОРЕТИЧЕСКАЯ

И МАТЕМАТИЧЕСКАЯ

ФИЗИКА

Том 127, № 3

июнь, 2001

(C) 2001 г.

Л. А. Калякин ${ }^{*}$

\section{ВОЗМУЩЕНИЕ РЕШЕНИЙ С ПОДВИЖНЫМИ ОСОБЫМИ ТОЧКАМИ}

Исследована задача Коши для нелинейного дифференциального уравнения второго порядка с малым параметром в случае, когда точное решение имеет степенную особенность и точка сингулярности зависит от малого параметра. Предложен способ асимптотической локализации особой точки с любым порядком точности, похожий на метод Крылова - Боголюбова. Вне особой точки построено асимптотическое разложение решения.

\section{1. ВВЕДЕНИЕ}

В настояшей работе рассматривается задача Коши для нелинейного обыкновенного дифференциального уравнения с малым параметром

$$
\begin{gathered}
U^{\prime \prime}+A\left(U, U^{\prime}, t\right)=\varepsilon B\left(U, U^{\prime}, t\right), \quad t>0, \quad 0<\varepsilon \ll 1, \\
\left.U\right|_{t=0}=u_{0},\left.\quad U^{\prime}\right|_{t=0}=v_{0}
\end{gathered}
$$

в случае, когда точное решение имеет степенную особенность $U(t ; \varepsilon)=\mathcal{O}\left(|T-t|^{-\nu}\right)$ при $t \rightarrow T<\infty(\nu=$ const $>0)$.

В теории возмушений, как правило, не исследуются задачи, решения которых содержат сингулярности. Широко распространенные термины “сингулярные" и "бисингулярные" возмушения [1]-[4] имеют отношение к особенностям коэффициентов асимптотики, но не к особенностям точных решений. В данной работе рассматривается ситуация, когда сингулярности имеются в точных решениях как возмушенной, так и невозмушенной задач. Подобные постановки обсуждаются не часто и обычно связываются с уравнениями в частных производных [5], [6]. Между тем наличие сингулярностей в решениях является характерной чертой многих нелинейных обыкновенных дифференциальных уравнений. Так, известные уравнения Пенлеве имеют богатые двухпараметрические наборы решений, одним из параметров которых является (подвижный) полюс [7]. Наличие полюсов в них обусловлено степенными нелинейностями. Мы рассматриваем несколько более общие уравнения (1) с гладкими функциям $A(u, v, t), B(u, v, t)$, которые

\footnotetext{
${ }^{*}$ Институт математики, Уфа, Россия. E-mail: klen@imat.rb.ru
} 
имеют степенное асимптотическое разложение на бесконечности при $|u|+|v| \rightarrow \infty$. При этом в общей ситуации решения имеют сингулярности, отличные от полюсов. Следует подчеркнуть, что задача рассматривается на действительной оси $t \in \mathbb{R}$, аналитическая структура решения в окрестности особых точек не выясняется и сходимость рядов не используется. В обсуждаемой задаче мы обходимся асимптотическими разложениями.

В качестве иллюстрации приведем пример возмущения уравнения Риккати, простейшего из уравнений, которые имеют сингулярные решения: $U^{\prime}=U^{2}+\varepsilon B(U)$. Невозмушенное решение с начальным условием $\left.U\right|_{t=0}=1$ имеет полюс первого порядка $U(t ; 0)=(1-t)^{-1}$. Свойства возмушенного решения $U(t ; \varepsilon)$ зависят от структуры возмущения. Например, в случае $B(u)=-u^{4}$ возмущенное решение не имеет сингулярностей. Если $B(u)=u^{4}$, то возмушенное решение имеет особую точку второго порядка:

$$
U(t ; \varepsilon)=\left(3 \varepsilon\left(T_{\varepsilon}-t\right)\right)^{-\frac{1}{3}}+\mathcal{O}\left(\left(T_{\varepsilon}-t\right)^{\frac{1}{3}}\right), \quad t \rightarrow T_{\varepsilon}=1-\frac{\sqrt{\varepsilon} \pi}{2}+\mathcal{O}(\varepsilon) .
$$

Наконец, при $B(u)=u$ возмущенное решение

$$
U=\varepsilon[\exp (-\varepsilon t)-1+\varepsilon \exp (-\varepsilon t)]^{-1}
$$

имеет полюс первого порядка в точке $T_{\varepsilon}=\varepsilon^{-1} \ln (1+\varepsilon)=1-\varepsilon / 2+\mathcal{O}\left(\varepsilon^{2}\right)$. Нетрудно убедиться, что таким свойством обладает решение при любом возмушении, которое на бесконечности имеет асимптотику в виде полинома второй степени $B(u)=b_{2} u^{2}+b_{1} u+$ $b_{0}+\mathcal{O}\left(u^{-1}\right),|u| \rightarrow \infty$. Задачи с такого типа возмущениями будут рассмотрены ниже для уравнений второго порядка. При изложении будем ориентироваться на примеры двух уравнений. Первое из них $U^{\prime \prime}-\left(U^{\prime}\right)^{2} / U-U^{3}=0$ представляет собой редукцию известного уравнения Лиувилля [8], [9] $u_{t t}-u_{x x}-\exp u=0$ и замечательно тем, что интегрируется в элементарных функциях: $U(t ; T, E)=E / \sin (E(T-t)) \forall E, T=$ const. Второй пример - первое уравнение Пенлеве $U^{\prime \prime}-6 U^{2}+t=0$, его решение представляет собой новую специальную функцию. Общим для обоих примеров является наличие двухпараметрических решений с подвижными полюсами.

\section{2. ИСХОДНЫЕ ОГРАНИЧЕНИЯ И РЕЗУЛЬТАТЫ}

В обшем случае рассматриваются уравнения с решениями, которые имеют особые точки алгебраического типа $U(t ; T, E)=\mathcal{O}\left(|T-t|^{-\nu}\right)$ с рациональным показателем $\nu=$ $p / q$, где $p, q \in \mathbb{Z}$. Введем подходящие классы функций для описания таких решений.

ОПРЕДЕЛЕНИЕ. Через $\mathcal{C}_{\lambda}^{\infty}=\mathcal{C}_{\lambda}^{\infty}(\Omega)$ будем обозначать линейное пространство функций трех переменных $U(t ; T, E)$, которые определены на множестве $\Omega=\{0 \leqslant t<T$, $(T, E) \in \Pi\}$, бесконечно дифференцируемы по $t$, непрерывны по $T, E$ и имеют асимптотическое разложение

$$
U(t ; T, E)=(T-t)^{-\lambda} U_{-\lambda}(T, E)+\sum_{\mu}(T-t)^{\mu} U_{\mu}(\ln |T-t| ; T, E), \quad t \rightarrow T .
$$


Счетный набор показателей $\mu \in(-\lambda, \infty)$ упорядочен и неограниченно возрастает: $\mu \rightarrow \infty$. В коэффициентах асимптотики допускается полиномиальная зависимость от логарифмов

$$
U_{\mu}(z ; T, E)=\sum_{i=0}^{\kappa} z^{i} U_{\mu, i}(T, E), \quad \kappa=\kappa(\mu)<\infty
$$

ЗАмЕчАниЕ. Все асимптотики считаются равномерными по $(T, E) \in \Pi$. Множество параметров П представляет собой замыкание ограниченной области из $\mathbb{R}^{2}$. Степени полиномов $\kappa(\mu)$ могут быть различными для разных функций. Для решений нелинейных уравнений вида (1) показатели $\mu$ представляют собой целочисленные комбинации $\mu=m \nu+n r+k, m, n, k \in \mathbb{Z}$, пары так называемых резонансных индексов $\nu, r$.

Исходные ограничения большей частью формулируются в терминах решения невозмушенного уравнения.

1. Предполагается, что невозмушенное уравнение $U^{\prime \prime}+A\left(U, U^{\prime}, t\right)=0$ имеет двухпараметрическое семейство решений $U \stackrel{0}{U}(t ; T, E)$ из класса $\mathcal{C}_{\nu}^{\infty}$ с подвижной особой точкой $T$ степени $\nu$ и со значениями параметров из области $(T, E) \in \Pi$. Решение невозмушенной задачи, фиксированное начальными данными (2), входит в такое семейство, и ему соответствуют параметры $\left(T_{0}, E_{0}\right) \in \Pi \backslash \partial \Pi$.

2. Вблизи особой точки имеет место асимптотическое разложение вида (4) с постоянным главным коэффициентом, который без ограничения общности можно считать положительным: $\stackrel{0}{U}-\nu=$ const $>0$. Функция $\stackrel{0}{U}(t ; T, E)$ и ее асимптотика считаются дважды непрерывно дифференцируемыми по параметрам $T, E$ и бесконечно дифференцируемыми по $t$, так что непрерывны и соответствующие смешанные производные.

3. Зависимость от двух произвольных параметров в невозмушенном решении предполагается существенной. Это означает, что определитель Вронского на паре функций $V_{T}=\partial_{T} \stackrel{0}{U}(t ; T, E), \quad V_{E}=\partial_{E} \stackrel{0}{U}(t ; T, E)$ отличен от нуля на множестве $\Omega$; на границе $t=T$ возможен нуль конечного порядка.

Исходные уравнения с функциями $A(u, v, t), B(u, v, t)$ определены в части фазовой плоскости $(u, v) \in \mathcal{D} \subset \mathbb{R}^{2}$ (зависимость от $t \in \mathbb{R}$ считается гладкой всюду). Уточнение условий на область $\mathcal{D}$ касается окрестности бесконечности. Ввиду асимптотики $\stackrel{0}{U}(t ; T, E)=(T-t)^{-\nu} \stackrel{0}{U}-\nu[1+o(1)]$ все семейство невозмушенных фазовых траекторий $\left(\stackrel{0}{U}, \stackrel{0}{U^{\prime}}\right)$ при $t \rightarrow T$ стремится к бесконечности, асимптотически приближаясь к параболе $v=u^{(\nu+1) / \nu} / \nu$. Поэтому область $\mathcal{D}$ не ограничена. Требование к ней состоит в том, что вместе с невозмушенной асимптотой в области $\mathcal{D}$ содержится некоторый криволинейный сектор, точнее, его неограниченная часть. В качестве границ такой неограниченной подобласти $\mathcal{D}_{\nu}$ возьмем отрезок прямой $u=c=$ const $>0$ и пару кривых, которые соответствуют функциям $U_{ \pm}(t)=(T-t)^{-\nu} \alpha_{ \pm},\left(0<\alpha_{-}<1<\alpha_{+}\right)$и представляют собой параболы $v=\left(\alpha_{ \pm} u\right)^{(\nu+1) / \nu} / \nu$. 
4. Таким образом, условие на область определения $\mathcal{D}$ :

$$
\mathcal{D}_{\nu} \equiv\left\{(u, v) \in \mathbb{R}^{2}:\left(\alpha_{-} u\right)^{\frac{\nu+1}{\nu}}<\nu v<\left(\alpha_{+} u\right)^{\frac{\nu+1}{\nu}} ; u>c\right\} \subseteq \mathcal{D} .
$$

5. Рассматриваются уравнения вида (1) с гладкими функциями $A(u, v, t), B(u, v, t)$, которые на бесконечности имеют разложение по целым степеням

$$
A(u, v, t)=\sum_{i, j=-m}^{\infty} a_{i, j}(t) u^{-i} v^{-j}, \quad B(u, v, t)=\sum_{i, j=-m}^{\infty} b_{i, j}(t) u^{-i} v^{-j}
$$

с конечным числом положительных степеней $(m=$ const $<\infty)$. Эти разложения считаются асимптотическими на бесконечности при $|u|+|v| \rightarrow \infty$ равномерно в секторе $\mathcal{D}_{\nu}$.

6. На показатели степеней накладываются ограничения, связанные с порядком особенности невозмущенного решения:

$$
\forall i, j: a_{i, j} \neq 0 \vee b_{i, j} \neq 0 \Longrightarrow-i \nu-j(\nu+1) \leqslant \nu+2
$$

ЗАмЕчАнИЕ. Для невозмущенного уравнения условие 6 и присутствие членов $\left\{a_{i, j} \neq 0:-i \nu-j(\nu+1)=\nu+2\right\}$ необходимы, чтобы сушествовало сингулярное решение $\stackrel{0}{U}(t ; T, E)=\mathcal{O}\left((T-t)^{-\nu}\right)$. Поэтому условие 6 , по сути дела, выделяет класс возмущений $B$, при которых гарантируется сохранение порядка особенности в возмущенном решении.

ТЕОРЕМА 1. Если выполнены условия 1-6, то справедливы утверждения:

I. Существует такое $\varepsilon_{0}>0$, что при всех $\varepsilon \in\left[0, \varepsilon_{0}\right]$ возмущенное уравнение (1) имеет двухпараметрическое семейство решений $U(t ; T, E ; \varepsilon)$, для каждого из которых граница $t=T$ представляет особую точку.

II. Возмущенное решение имеет асимптотическое разложение

$$
U(t ; T, E ; \varepsilon)=\sum_{n=0}^{\infty} \varepsilon^{n} \stackrel{n}{U}(t ; T, E), \quad \varepsilon \rightarrow 0
$$

с коэффициентами $\stackrel{n}{U}(t ; T, E) \in \mathcal{C}_{\nu}^{\infty}(\Omega)$. Это разложсение асимптотическое в равномерной метрике $C(\Omega)$ с весом $(T-t)^{\nu}$.

III. При всех $\varepsilon \in\left[0, \varepsilon_{0}\right]$ начальная задача для возмущенного уравнения (1), (2) имеет единственное решение, которое вырахсается через семейство $U(t ; \varepsilon)=$ $U(t ; T(\varepsilon), E(\varepsilon) ; \varepsilon)$ посредством гладких функиий $T=T(\varepsilon), E=E(\varepsilon)$.

IV. Для особой точки $T(\varepsilon)$ и для параметра $E(\varepsilon)$ имеют место асимптотические разложения

$$
T(\varepsilon)=\sum_{n=0}^{\infty} \varepsilon^{n} T_{n}, \quad E(\varepsilon)=\sum_{n=0}^{\infty} \varepsilon^{n} E_{n}, \quad \varepsilon \rightarrow 0
$$


СЛЕДСТВИЕ. На основе построенных рядов возможна асимптотическая аппроксимация решения равномерно вне сколь угодно узкой (по порядку в) окрестности особой точки, так что $\forall N \exists M$ = const:

$$
\left|U(t ; \varepsilon)-\sum_{n} \varepsilon^{n} \stackrel{n}{U}\left(t ; T_{N}(\varepsilon), E_{N}(\varepsilon)\right)\right| \leqslant M \varepsilon^{N}, \quad 0 \leqslant n \leqslant(\nu+1) N
$$

равномерно для $\forall t \in\left[0, T(\varepsilon)-\varepsilon^{N}\right]$ с соответствующей аппроксимачией параметров: $T(\varepsilon)=T_{N}(\varepsilon)+\mathcal{O}\left(\varepsilon^{N+1}\right), \quad E(\varepsilon)=E_{N}(\varepsilon)+\mathcal{O}\left(\varepsilon^{N+1}\right)$.

\section{3. ПОСТРОЕНИЕ АСИМПТОТИЧЕСКОГО РЕШЕНИЯ}

В общепринятой классификации рассматриваемая задача относится к задачам с сингулярным возмущением. Прямой ряд теории возмущений с не зависяшими от $\varepsilon$ коэффициентами

$$
U(t ; \varepsilon)=\sum_{n=0}^{\infty} \varepsilon^{n} \stackrel{n}{U}(t), \quad \varepsilon \rightarrow 0
$$

не является асимптотическим вблизи невозмушенной особой точки при $\left|t-T_{0}\right| \leqslant \mathcal{O}(\varepsilon)$. Это можно наглядно продемонстрировать на примере уравнения Риккати, для которого в прямом разложении возмушенного решения (3) имеются особенности растушего порядка $U_{n}(t)=\mathcal{O}\left((1-t)^{-n-1}\right)$, связанные с разложением функции типа $(1-t-\varepsilon)^{-1}$. Усиление особенностей делает непригодной асимптотику в такой форме вблизи точки $T_{0}$ на расстояниях порядка $\mathcal{O}(\varepsilon)$. Проблемы возникают из-за зависимости от $\varepsilon$ положения возмушенного полюса $T(\varepsilon)$, для которого, кстати, из прямого разложения невозможно получить асимптотику при $\varepsilon \rightarrow 0$.

Предлагаемый нами способ построения формального асимптотического решения $(\Phi \mathrm{AP})$ использует известные идеи, которые обычно связываются с именами Крылова, Боголюбова и Кузмака [10], [11]. ФАР строится в виде ряда (5), в котором для главного члена используется обшее решение предельного уравнения: $\stackrel{0}{U}=\stackrel{0}{=}(t ; T, E)$. Неизвестными являются старшие поправки $\stackrel{n}{U}=\stackrel{n}{U}(t ; T, E), n \geqslant 1$, как функции от $t, T, E$ и параметры $T, E$ как функции от $\varepsilon$. Основная идея состоит в допушении зависимости от $\varepsilon$ параметров $T, E$. Для них строятся свои асимптотические разложения при $\varepsilon \rightarrow 0$ в виде степенных рядов (6).

Для поправок выписывается рекуррентная система линеаризованных уравнений:

$$
\stackrel{n}{U^{\prime \prime}}+A_{1}(t ; T, E) \stackrel{n}{U^{\prime}}+A_{0}(t ; T, E) \stackrel{n}{U}=\stackrel{n}{F}(t ; T, E) .
$$

Коэффициенты здесь выражаются через решение невозмушенного уравнения: $A_{1}=$ $\partial_{v} A\left(\stackrel{0}{U}, \stackrel{0}{U^{\prime}}, t\right), A_{0}=\partial_{u} A\left(\stackrel{0}{U}, \stackrel{0}{U^{\prime}}, t\right)$. Правые части зависят от предыдущих приближений и вычисляются с использованием коэффициентов Тейлора функций $A(u, v, t), B(u, v, t)$, 
например, при $u=\stackrel{0}{=}, v \stackrel{0}{U^{\prime}}$

$$
\begin{aligned}
F_{1}= & B(u, v, t) \\
F_{2}= & \partial_{u} B\left(u, v, \stackrel{1}{U}+\partial_{v} B\left(u, v, \stackrel{1}{U^{\prime}}-\frac{1}{2} \partial_{u}^{2} A\left(u, v, \stackrel{1}{U^{2}-}\right.\right.\right. \\
& \left.-\frac{1}{2} \partial_{v}^{2} A(u, v, t) \stackrel{1}{U^{\prime}}\right)^{2}-\partial_{u} \partial_{v} A(u, v, t) \stackrel{1}{U} U^{\prime}
\end{aligned}
$$

Решения $\stackrel{n}{U}$ вьписываются в явном виде в терминах главного члена $\stackrel{0}{U}$ и потому зависят от параметров $T, E$. Начальные условия при этом не используются. Дополнительные ограничения на поправки $\stackrel{n}{U}$ состоят в требовании отсутствия у них сингулярностей более сильных, чем в главном члене. Это требование аналогично секулярному условию в задачах об усреднении быстрых колебаний [10], [11].

Таким образом строится семейство ФАР, зависящее от двух параметров $T, E$. На заключительном этапе эти параметры определяются как функции от $\varepsilon$ и строится их асимптотика при $\varepsilon \rightarrow 0$ исходя из начальных условий. Реализуя указанную процедуру, докажем следующее утверждение.

Теорема 2. Если выполнены требования теоремы 1, то рекуррентная система линеаризованных уравнений (7) разрешима в классе $\mathcal{C}_{\nu}^{\infty}$.

Доказательство основано на использовании пары решений однородного уравнения, в качестве которых берутся производные по параметрам: $V_{T}(t ; T, E)=\partial_{T} \stackrel{0}{U}$, $V_{E}(t ; T, E)=\partial_{E} \stackrel{0}{U} . \quad$ Функции $V_{T}, V_{E}$ обладают свойством $V_{T} \in \mathcal{C}_{\nu+1}^{\infty}, \quad V_{E} \in \mathcal{C}_{-r}^{\infty}$, $-\nu<r<\infty$, и для них можно записать асимптотические формулы при $t \rightarrow T$

$$
V_{T}(t ; T, E)=(T-t)^{-\nu-1} \stackrel{0}{U}_{-\nu}[-\nu+o(1)], \quad V_{E}(t ; T, E)=(T-t)^{r} V_{r}[1+o(1)]
$$

с вполне определенными главными членами и с коэффициентом $V_{r} \neq 0$.

ЗАМЕчАнИЕ. Индекс $r$ определяет в асимптотическом разложении для невозмущенного решения $\stackrel{0}{U}$ слагаемое $(T-t)^{r} \stackrel{0}{U} r(T, E)$, главное среди тех, которые содержат произвольньй параметр ${ }^{1)}$ (см. пример с уравнением Лиувилля).

Вронскиан $W\left(V_{T}, V_{E}\right) \equiv w(t ; T, E)$, как функция от $(t ; T, E)$, принадлежит классу $\mathcal{C}_{\nu-r+2}^{\infty}$ с асимптотикой

$$
w(t ; T, E)=-\nu(\nu+1-r)(t-T)^{-\nu+r-2} V_{r} \stackrel{0}{U_{-\nu}}[1+o(1)]
$$

Поскольку вронскиан не обращается в нуль при $(t, T, E) \in \Omega$, а на границе $t=T$ допускается нуль лишь конечного порядка, то из этой асимптотики вытекает конечность

\footnotetext{
${ }^{1)}$ Без ограничения общности можно считать, что этот коэффициент (называемый иногда резонансным) совпадает с параметром $\stackrel{0}{U} r=E$, так что $V_{r}=\partial_{E} \stackrel{0}{U}_{r}=1$.
} 
резонансного показателя $-\nu<r<\infty$. Решение неоднородного линеаризованного уравнения выписывается в явной форме

$$
\begin{aligned}
U(t ; T, E)= & V_{E}(t ; T, E) \int_{t_{0}}^{t} V_{T}(\zeta ; T, E) \frac{F(\zeta ; T, E)}{w(\zeta ; T, E)} d \zeta- \\
& -V_{T}(t ; T, E) \int_{T}^{t} V_{E}(\zeta ; T, E) \frac{F(\zeta ; T, E)}{w(\zeta ; T, E)} d \zeta \equiv \mathbf{I}[F], \quad t_{0}=\text { const. }
\end{aligned}
$$

Эта формула определяет линейный интегральньй оператор $\mathbf{I}[F]$, для которого можно доказать ограниченность на подходяшей паре функциональных пространств. Пока же достаточно установить следуюшее свойство.

ЛЕмма 1. Если $F \in \mathcal{C}_{\nu+2}^{\infty}$, mo $\mathbf{I}[F] \in \mathcal{C}_{\nu}^{\infty}$.

Доказательство основано на интегрировании асимптотических разложений вида (4), которые получаются для подынтегральных выражений в (8).

Для завершения доказательства теоремы 2 надо проверить, что правые части $\stackrel{n}{F}$, которые возникают в рекуррентной системе линеаризованных уравнений (7), всегда принадлежат классу $\mathcal{C}_{\nu+2}^{\infty}$, если только предыдушие решения $\stackrel{k}{U}, k<n$, были из $\mathcal{C}_{\nu}^{\infty}$. Решающую роль в сохранении порядка особенности $\nu$ играет условие 6 , которое ограничивает степень роста нелинейностей на бесконечности. Теорема 2 доказана.

Заключительный этап построения $\Phi$ АР состоит в удовлетворении начальных условий (2) за счет произвола в параметрах $(T, E)$. Подстановка $\Phi$ АР (5) в соотношения (2) приводит к паре алгебраических уравнений для $T, E$, которые решаются асимптотически. Задача сводится к определению коэффициентов $T_{n}, E_{n}$ в рядах $(6)$, для которых можно получить рекуррентную систему уравнений. При этом в главном порядке уравнения выполняются в силу выбора параметров невозмушенного решения: $\stackrel{0}{U}\left(0 ; T_{0}, E_{0}\right)=$ $u_{0}, \stackrel{0}{U^{\prime}}\left(0 ; T_{0}, E_{0}\right)=v_{0}$. Последующие приближения определяются из рекуррентной системы линеаризованных уравнений

$$
V_{E}\left(0 ; T_{0}, E_{0}\right) E_{n}+V_{T}\left(0 ; T_{0}, E_{0}\right) T_{n}=u_{n}, \quad V_{E}^{\prime}\left(0 ; T_{0}, E_{0}\right) E_{n}+V_{T}^{\prime}\left(0 ; T_{0}, E_{0}\right) T_{n}=v_{n}
$$

с ненулевым определителем. Правые части вычисляются через предыдущие приближения и известны настолько, насколько известны соответствующие поправки в ФАР (5). Тем самым доказана

ЛЕмма 2. Если для функиий $T(\varepsilon), E(\varepsilon)$ коэффициенты асимптотических разложений (6) удовлетворяют уравнениям (9), то ФАР (5) с соответствующими параметрами $T=T(\varepsilon), \quad E=E(\varepsilon)$ асимптотически удовлетворяет начальным условиям (2). 
ЗАМЕЧАнИЕ К НЕЕДИНСТВЕНности ФАР. Неединственность в конструкции ФАР $(5),(6)$ связана с гладкой частью зависимости коэффициентов от $\varepsilon$. Такая зависимость входит посредством параметра $E=E(\varepsilon)$ и частично посредством гладкой зависимости от параметра $T=T(\varepsilon)$. После частичного либо полного переразложения по $\varepsilon$ получаются ряды такого же типа (с новыми коэффициентами $U_{n}, E_{n}$ ). Каждая пара рядов представляет собой ФАР задачи (1), (2). Поскольку все такие пары рядов асимптотически эквивалентны, то их неединственность не играет роли: разность отрезков двух рядов для $U$ с подстановкой соответствуюших асимптотик для $E$ не превьшает отброшенных членов по порядку величины $\varepsilon$.

Кроме того, неединственность можно обнаружить на этапе формальных построений в коэффициентах $\stackrel{n}{U}$. Она связана с произволом в выборе нижнего предела в первом интеграле (8), так что на каждом шаге возможна добавка одного из решений однородного уравнения $^{2)} \beta V_{E}=\beta \partial_{E} U_{0}(t ; T, E)$ с произвольной константой $\beta$. Произвол, естественно, проявляется при решении начальных уравнений (9) для $E_{n}, T_{n}$. Критическим для всей конструкции является вопрос об однозначности коэффициентов $T_{n}$, которые определяют асимптотику сингулярной точки $T(\varepsilon)$. При желании можно доказать однозначность ряда для $T$ и асимптотическое совпадение всех рядов для $U$, которые могут быть получены таким способом. Аналогично [12] можно установить, что проблема сводится к изменению в выборе функции $E=E(\varepsilon)$ с переразложением ряда для $U$, как это описано выше. Однако мы решаем этот вопрос иначе, доказывая единственность точного решения исходной задачи (1), (2), для которого любое из построенных ФАР представляет асимптотическое разложение. Такое доказательство описано в следующем разделе.

\section{4. ОБОСНОВАНИЕ АСИМПТОТИКИ}

Обоснование проводится в два этапа. На первом этапе для возмушенного уравнения (1) строится семейство точных решений $U(t ; T, E ; \varepsilon)$ с подвижной особой точкой $T$. При этом все три параметра $T, E \in \Pi, \varepsilon \in\left[0, \varepsilon_{0}\right]$ независимы. На втором этапе подходящие значения $T=T(\varepsilon), E=E(\varepsilon)$ выбираются исходя из требования выполнения начальных условий $(2)$.

Точное решение уравнения (1) ишется в виде суммы конечного отрезка $U_{N}$ построенного $\Phi А Р(5)$ и неизвестного остатка $Y$ :

$$
U(t ; T, E ; \varepsilon)=U_{N}(t ; T, E ; \varepsilon)+\varepsilon^{N} Y(t ; T, E ; \varepsilon) .
$$

Функция $U_{N}$ при подстановке в уравнение (1) дает невязку порядка $\mathcal{O}\left(\varepsilon^{N+1}\right)$ в равномерной метрике с весом $\mathcal{O}\left((T-t)^{\nu}\right)$. В дифференциальном уравнении для остаточного члена $Y$, которое получается из (1), можно идентифицировать главную часть в виде линеаризованного оператора

$$
Y^{\prime \prime}+A_{1}(t ; T, E) Y^{\prime}+A_{0}(t ; T, E) Y=-\varepsilon F_{N}(t ; T, E ; \varepsilon)+\varepsilon \mathbf{H}[Y ; \varepsilon] .
$$

\footnotetext{
2) Как видим, требование отсутствия сильных сингулярностей не достаточно для единственности решения линеаризованного уравнения.
} 
При этом в нелинейном операторе $\varepsilon \mathbf{H}$ так же, как в невязке $\varepsilon F_{N}$, можно выделить малый множитель, если определить $\mathbf{H}$ в виде

$$
\mathbf{H}[Y ; \varepsilon] \equiv H_{B}\left(Y, Y^{\prime}, U_{N}, U_{N}^{\prime}, t ; \varepsilon\right)-H_{A}\left(Y, Y^{\prime}, U_{N}, U_{N}^{\prime}, \stackrel{0}{U} \stackrel{0}{U^{\prime}}, t ; \varepsilon\right)
$$

через функции

$$
\begin{aligned}
& H_{B}(y, z, u, v, t ; \varepsilon) \equiv \frac{1}{\varepsilon^{N}}\left[B\left(u+\varepsilon^{N} y, v+\varepsilon^{N} z, t\right)-B(u, v, t)\right] \\
& H_{A}\left(y, z, u, v, u^{0}, v^{0}, t ; \varepsilon\right) \equiv \frac{1}{\varepsilon^{N+1}}\left[A\left(u+\varepsilon^{N} y, v+\varepsilon^{N} z, t\right)-A(u, v, t)-\right. \\
& \left.\quad-\varepsilon^{N} \partial_{u} A(u, v, t) y-\varepsilon^{N} \partial_{v} A(u, v, t) z\right]+ \\
& \quad+\frac{1}{\varepsilon}\left[\partial_{u} A(u, v, t)-\partial_{u} A\left(u^{0}, v^{0}, t\right)\right] y+\frac{1}{\varepsilon}\left[\partial_{v} A(u, v, t)-\partial_{v} A\left(u^{0}, v^{0}, t\right)\right] z
\end{aligned}
$$

при $y=Y, z=Y^{\prime} ; u=U_{N}, v=U_{N}^{\prime} ; u^{0}=\stackrel{0}{U}, v^{0}=\stackrel{0}{U^{\prime}}$. Заметим, что эти функции состоят из остаточных членов разложений Тейлора для функций $A(u, v, t), B(u, v, t)$ в точках $(u, v)$ или $\left(u^{0}, v^{0}\right)$, а потому являются гладкими по всем переменным в области определения $\left(u^{0}, v^{0}\right),(u, v),\left(u+\varepsilon^{N} y, v+\varepsilon^{N} z\right) \in \mathcal{D} \forall t, \varepsilon$.

Если речь идет о гладких решениях, то не вызьвает сомнения разрешимость уравнения (10) с соответствуюшими начальными данными. Для решений с особенностями основные проблемы возникают с сушествованием и оценкой остаточного члена на промежутке вблизи особой точки $t \in\left[t_{0}, T\right)$. В этом случае вводятся подходящие весовые пространства, в которых затем реализуется метод сжимающих отображений.

\section{5. ПРИМЕР}

Рассмотрим возмушение редуцированного уравнения Лиувилля

$$
U^{\prime \prime}-\frac{\left(U^{\prime}\right)^{2}}{U}-U^{3}=\varepsilon\left(\frac{U^{\prime}}{U}\right)^{2}
$$

при начальных условиях $\left.\left(U, U^{\prime}\right)\right|_{t=0}=(1,0)$. Используя обозначение для фазовой функции $z=(T-t) E$, первые два члена асимптотики решения можно записать в форме

$$
\begin{aligned}
U(t ; T, E) & =\frac{1}{\sin z}\left\{E+\varepsilon\left[\frac{\sin z-z \cos z}{\sin z}\left(\frac{1+\sin ^{2}(T E)}{\sin ^{2}(T E)}-\frac{1+\sin ^{2} z}{\sin ^{2} z}\right)-\right.\right. \\
- & \left.\left.\frac{\cos z}{\sin z}\left(\frac{z}{\sin z}+z \sin z+2 \cos z-3\right)\right]+\mathcal{O}\left(\varepsilon^{2}\right)\right\}, \quad \varepsilon \rightarrow 0, \quad \forall t \in[0, T) .
\end{aligned}
$$

Параметры имеют асимптотические разложения

$$
T=\frac{\pi}{2}-\varepsilon(\pi-3)+\mathcal{O}\left(\varepsilon^{2}\right), \quad E=1+\mathcal{O}\left(\varepsilon^{2}\right) .
$$

Таким образом, под действием малого возмушения полюс сдвигается на величину порядка возмущения. Оценка остаточного члена в формуле (11) равномерная на всем промежутке $0 \leqslant t<T$, поскольку сингулярность вынесена в виде обшего (весового) множителя. 


\section{6. ЗАКЛЮЧЕНИЕ}

Асимптотика параметров $T, E$ главного члена решения извлекается из начальных уравнений (9). В общем случаепервая поправка в сдвиге сингулярной точки $T(\varepsilon)=T_{0}+$ $\varepsilon T_{1}+\mathcal{O}\left(\varepsilon^{2}\right)$ вычисляется через интеграл от возмущения:

$$
T_{1}=-\int_{0}^{T} V_{E}\left(t ; T_{0}, E_{0}\right) \frac{B\left(\stackrel{0}{U}, \stackrel{0}{U^{\prime}}, t\right)}{w\left(t ; T_{0}, E_{0}\right)} d t
$$

в терминах невозмушенного решения $\stackrel{0}{U}\left(t ; T_{0}, E_{0}\right)$, его производных $V_{E}=\partial_{E} \stackrel{0}{U}, V_{T}=$ $\partial_{T} \stackrel{0}{U}$ и вронскиана $w=W\left(V_{T}, V_{E}\right)$. Если первая поправка в решении $U=\stackrel{0}{U}(t ; T, E)+$ $\stackrel{1}{U}(t ; T, E)+\mathcal{O}\left(\varepsilon^{2}\right)$ вьписывается по формуле $(8)$ с $t_{0}=0$, то первая поправка в параметре $E$ оказывается нулевой: $E=E_{0}+\mathcal{O}\left(\varepsilon^{2}\right)$. Этот параметр будет совпадать с невозмушенным значением $E_{0}$ во всех порядках при подходяшем выборе старших поправок $\stackrel{n}{U}$. В задачах о возмушении уравнений Пенлеве асимптотические формулы выписываются в терминах соответствующих трансцендентов Пенлеве.

Благодарности. Работа выполнена при поддержке РФФИ (гранты № 00-01-00663, 00-15-96038) и фонда INTAS (№ 99-1068).

\section{Список литературы}

[1] Е.Ф. Мищенко, Н. Х. Розов. Дифференциальные уравнения с малым параметром и релаксационные колебания. М.: Наука, 1975.

[2] A. Найфә. Методы возмушений. М.: Мир, 1984.

[3] А. М. Ильин. Согласование асимптотических разложений решений краевых задач. М.: Наука, 1989.

[4] А.Б. Васильева, В.Ф. Бутузов. Асимптотические методы в теории сингулярных возмушений. М.: Высшая школа, 1990.

[5] В. П. Маслов. Комплексный метод ВКБ в нелинейных уравнениях. М.: Наука, 1977.

[6] Л. А. Калякин. ТМФ. 1999. Т. 118. № 3. С. 390-397.

[7] В.И. Громак, Н. А. Лукашевич. Аналитические свойства решений уравнений Пенлеве. Минск: Университетское изд-во, 1990.

[8] J. Liouville. J. Math. Pure Appl. 1853. V. 18. P. 71-74.

[9] А.К. Погребков, М. К. Поливанов. Тр. МИАН. 1987. Т. 176. С. 86-96.

[10] Н. М. Крылов, Н. Н. Боголюбов. Исследование продольной устойчивости аэроплана. М.-Л.: Гос. авиац. и автотракт. изд-во, 1932.

[11] Г. Е. Кузмак. ПММ. 1959. Т. 23. № 3. С. 515-526.

[12] L. A. Kalyakin. Russian J. Math. Phys. 1998. V. 5. P. 447-458. 